Rhode Island College

Digital Commons @ RIC

$5-1-2013$

\title{
Intra-Femoral Sheath Time: Does it Increase a Patient's Risk for Developing a Hematoma?
}

Colleen O'Brien Planchon

Rhode Island College

Follow this and additional works at: https://digitalcommons.ric.edu/etd

Part of the Nursing Commons

\section{Recommended Citation}

Planchon, Colleen O'Brien, "Intra-Femoral Sheath Time: Does it Increase a Patient's Risk for Developing a Hematoma?" (2013). Master's Theses, Dissertations, Graduate Research and Major Papers Overview. 222. https://digitalcommons.ric.edu/etd/222

This Major Paper is brought to you for free and open access by the Master's Theses, Dissertations, Graduate Research and Major Papers at Digital Commons @ RIC. It has been accepted for inclusion in Master's Theses, Dissertations, Graduate Research and Major Papers Overview by an authorized administrator of Digital Commons @ RIC. For more information, please contact digitalcommons@ric.edu. 


\title{
INTRA-FEMORAL SHEATH TIME:
}

DOES IT INCREASE A PATIENT'S RISK FOR DEVELOPING A HEMATOMA?

\author{
by
}

Colleen O'Brien Planchon

A Major Paper Submitted in Partial Fulfillment

of the Requirements for the Degree of

Master of Science in Nursing

in

The School of Nursing

Rhode Island College

2013 


\begin{abstract}
Despite advancements in technology and medication therapy, coronary artery disease continues to remain the number one cause of death. Treatment for coronary artery disease requires life style modifications, medication therapy, percutaneous coronary interventions, and sometimes coronary artery bypass surgery. Though percutaneous coronary interventions are considered safe and are commonly occurring procedures, there are still risks for complications. One of the most frequently occurring complications is hematoma of the femoral artery site. The purpose of this study was to determine if there was an increased incidence of hematomas in individuals undergoing percutaneous coronary intervention with associated sheath times of less than five hours (Group A) as compared to sheath time of greater than five hours (Group B). The study used a retrospective, two group design and was conducted at a tertiary care center that performs over 1500 PCI's annually. Inclusion criteria included adults over the age of 18 who were hemodynamically stable, had no known bleeding disorders, and were punctured once at the femoral artery to perform the percutaneous intervention. Two hundred fifty medical records were reviewed utilizing a data collection tool designed by the researcher. Total sample size was 55, 21 in Group A and 34 in Group B. Six hematomas were documented as occurring, but were not reportable based on the ACC definition. Hematomas were documented in the medical record using the terms "small", "medium," and "large" versus an objective measurement. No hematomas were identified using the ACC definition, which was the measurement standard for this research hospital. Sheath times in Group A averaged 4.02 hours, and group B 7.42 hours. There was a higher incidence of descriptive hematoma that did not meet the criteria of this study in Group B. Results call for recommendations of on-going documentation of post procedure hematomas using a standardized, reliable, and valid measuring tool. APRN's can be
\end{abstract}


instrumental in implementing change in patient care, hospital policy and on the national level by assuring that hematomas are accurately and consistently documented. Further research is indicated related to sheath times and potential associated negative outcomes.

Key words: Percutaneous coronary interventions, hematomas, femoral artery, sheath times, complications. 


\section{Table of Contents}

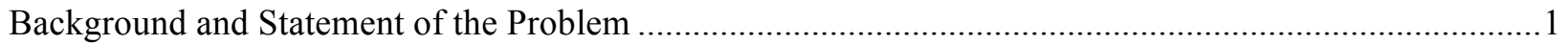

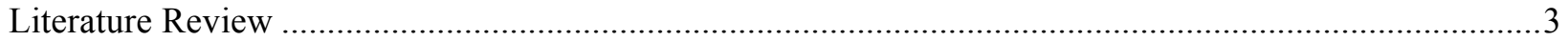

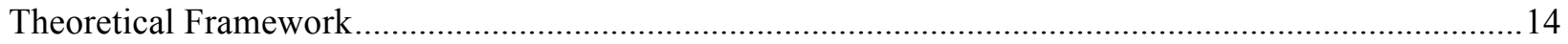

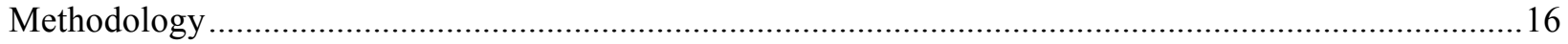

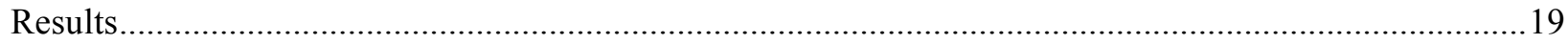

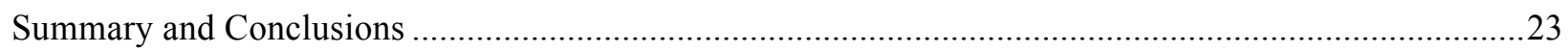

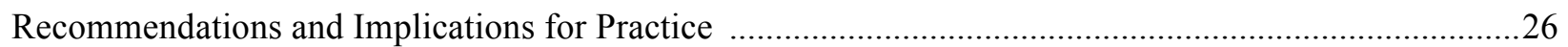

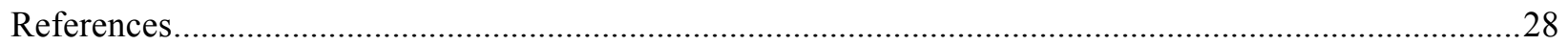

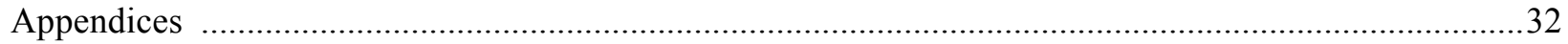




\section{Background and Statement of the Problem}

Cardiac disease remains the number one cause of death in persons over the age of 75 , and is ranked the number two cause of death in persons aged 25-74. In the past 15 years, advancements in technology and medicine have led to a significant increase in the number of cardiac catheterizations and percutaneous coronary interventions (PCIs) that have been performed. These include a broad category of procedures including angioplasty, placement of stents, artherectomy, laser treatment, cutting angioplasty, and brachytherapy (Shoulders-Odom, 2008). In the year 2006, there were over 1,300,000 PCI procedures performed in the United States (US). These numbers are projected to increase with the aging of the population (American Heart Association [AHA], 2009). Due to the increased proliferation of PCI procedures, hospitals capable of performing PCIs in the US have increased from 920 hospitals in 1993 to 1316 in 2004. PCIs receive generous reimbursement rates, and this, along with decreased federal regulatory processes, has led to the considerable increase in hospitals performing PCI procedures in the US (Girotra \& Cram, 2012).

The state of Rhode Island (RI) is approximately 1,045 square miles with a population of 1,052, 067 (US Census Bureau, 2010). Rhode Island is ranked $24^{\text {th }}$ in the incidence of heart disease in the US, and in 2009 heart disease was listed as the number one cause of death in RI, at 2,411 individuals (National Center for Health Statistics, 2010). The state has 13 hospitals, of which two are psychiatric hospitals. Of the remaining 11, eight are capable of performing cardiac catheterizations and three are licensed to perform PCIs (RI Department of Health, 2011).

Cardiac catheterization, an invasive procedure, is commonly used diagnostically and therapeutically for evaluation of patients with symptomatic coronary artery disease (CAD) (Cosman, Arthur, \& Natarajan, 2011). When therapy is required for treatment of the CAD, a 
PCI may be indicated. A PCI is performed during a cardiac catheterization procedure, at which time a catheter with a balloon is advanced to the site of the lesion and a stent, a hollow mesh tube which is left in place to attempt to keep the vessel patent, is deployed. Alternatively, a pertcutaneous transluminal coronary angioplasty (PTCA) may be performed, in which the balloon catheter is used to push the plague to the sides of the artery (McConnell et al., 2012).

As an experienced nurse working in a diagnostic cardiac catheterization lab, the author has noted anecdotally that approximately $20 \%$ of patients require travel to another facility in order to undergo the PCI. This requires that the sheath that was inserted into a major blood vessel in order to perform the catheterization must remain in place. There is some suggestion in the literature that the amount of time that a sheath remains in place may be associated with an increased risk of complications, including hematoma formation. For patients that need to be transferred to another facility, transport time, availability of paramedic staffed ambulances, and arranging staff to travel with the patient impact the total time that the sheath remains in place. In addition, a hemodynamically, asymptomatic patient who is transferred for PCI may be triaged to the end of the catheterization schedule, or postponed overnight, with the sheath remaining in place for an extended period of time. Even in circumstances where the PCI is performed within one institution, various factors impact the total time of the procedure and sheath time, including the practitioners' schedule, the institutions' schedule, and or the institutions' capability.

The purpose of this study was to determine if there was an increased incidence of hematomas in individuals undergoing percutaneous coronary intervention with associated sheath times of greater than five hours as compared to sheath time of less than five hours. 


\section{Literature Review}

A review of the literature was conducted utilizing search engines Pub Med, and CINAHL databases. Key words searched and combined included cardiac catheterization, percutaneous coronary intervention, femoral complications, femoral sheaths, hematoma, and risk factors for vascular complications. Literature from the years 2000 to 2013 was searched. Key literature will be presented and discussed.

Approximately 80,000,000 American adults have one or more types of cardiovascular disease (CVD), which includes hypertension (HTN), coronary artery disease (CAD), heart failure (HF), stroke, and congenital cardiovascular defects. Of the total, 38,100,000 are estimated to be age 60 years or older. Approximately 2,400 Americans die every 37 seconds from CVD (AHA, 2009). Coronary artery disease (CAD) is a major cause of death and disability in industrialized countries. CAD mortality rates have declined over the past four decades in the US, but still remain the number one cause of death (Rosamond et al., 2008 e25). CAD is responsible for about one-third of all deaths in individuals over age 35 , and it has been estimated that nearly onehalf of all middle-aged men and one-third of middle-aged women in the US will develop some manifestation of coronary artery disease (Lloyd-Jones et al., 2010).

\section{Epidemiology of Coronary Artery Disease}

Understanding of the pathophysiology of CAD has evolved throughout the $20^{\text {th }}$ century. Once thought to be singularly a cholesterol building disease, the epidemiology of CAD has shifted to a complex interaction of risk factors and an inflammatory immune response within an arterial lumen. In individuals with dyslipidemia, or concurrently with hypertension, when the arterial endothelium encounters certain bacteria, vasoconstrictor hormones augment the production of adhesion molecules that promote sticking of blood leukocytes to the inner surface 
of the arterial wall. What occurs next is highly related to the emerging risk factors for atherosclerosis, and the individual's pathophysiological response. Risk factors include, but are not limited to, high fat intake, obesity, hypertension, smoking, dyslipedemia, and genetics. Once the changes begin to occur in the arterial wall, the blood lymphocytes, mainly phagocytes, and $\mathrm{T}$ cells, activate the inflammation cascade within the arterial wall and CAD changes commence. As this process continues to synthesize in the arterial lumen, other physiological changes and mediators continue to occur over time. When the lumen completes the inflammation process, calcium deposits form within the lumen and remain there until the individual suffers an event such as a clot interacting in the narrow lumen (Libby \& Theroux, 2005).

\section{Overall Management of Coronary Artery Disease}

The management of CAD consists of lifestyle modification, medication therapy, and surgical interventions. Management begins with patient education by providing individuals with preventative strategies to decrease the risk of developing CAD. Teaching and guidance regarding modification of an individual's risk factor may include diet and exercise, weight modification, smoking cessation, and blood pressure control. If modifications fail to improve an individual's risk, medication therapy is prescribed (Smith Jar et al., 2006).

Medication therapy is aimed at reducing the individual's modifiable risk factors that have been shown to be a catalyst for development of CAD. Therapy approaches focus on cholesterol and blood pressure management. Target blood pressure and cholesterol values are based on the American College of Cardiology and the American Heart Association guidelines and the Joint

National Committee on Prevention, Detection, Evaluation and Treatment of High Blood Pressure (JNC 7); (Smith Jar et al., 2006; US Department of Health and Human Services [USDHHS], 2008) 
The recommendations include total cholesterol below $200 \mathrm{mg} / \mathrm{dl}$ with low-density lipoprotein (LDL) below $100 \mathrm{mg} / \mathrm{dl}$, high- density lipoprotein (HDL) greater or equal to 60 $\mathrm{mg} / \mathrm{dl}$, and triglycerides less than $150 \mathrm{mg} / \mathrm{dl}$. When cholesterol values fail to fall within these ranges a statin, or a lipid lowering medication, is prescribed based on the individual's results. Maintaining systolic blood pressure (SBP) less than 120-128, and diastolic of less than 80-89 $\mathrm{mm} \mathrm{Hg}$, are essential components in the management of hypertension (Lichtenstien et al., 2006) Medication therapy begins with a thiazide diuretic, which is a medication targeted at inhibiting resorption of sodium and chloride ions at the distal convoluted tubules in the kidneys. If thiazide therapy is not successful in achieving those desirable BP results, additional medications such as an angiotensin-converting enzyme inhibitor (ACE), and/or an angiotensin receptor blocker (ARB) should be added. Other medications that might be used, based on the individuals CAD symptoms, include aspirin, nitrates (nitroglycerin), beta-blockers, and calcium channel blockers (USDHHS, 2008).

If lifestyle modifications and medical management fail to prevent symptoms of CAD from prevailing and progressing, surgical intervention including, PCIs or coronary artery bypass surgery, are the final therapy options. Patients requiring intervention, whether it is PCI or bypass surgery, can be grouped into three categories: individuals who are limited in their activities due to symptoms not responding to medical therapy; individuals whose anatomy of target vessels have been proven benefited by revasculization; and those who desire bypass surgery or intervention for an improved quality of life in which the are not achieve by medications alone (Alderman et al., 2004). 


\section{Vascular Complications of Interventional Treatment}

Cardiac catheterizations and PCIs are considered routine procedures with a low threshold for serious complications. The rate of complications for diagnostic catheterization is no more than $1 \%$, and the complication rate associated with PCI is 3\% Complications that can disturb a patient's post procedure recovery, cause pain, and an increase in length of stay include vascular complications such as hematoma, pseudoaneurysm, arteriovenous fistula, peripheral artery occlusion, dissections, and retroperitoneal bleeding (Cale \& Constantino, 2012). Vascular complications alone have been reported from $0.1 \%$ to up to $61 \%$, depending on the definition of complications and covariates (Dumont, Keeling, Bourguignon, Sarembock, \& Turner, 2006).

The femoral artery site is commonly used for access over other arteries, and is recognized as the most common site of vascular complications (Patel et al., 2010). Significant vascular complications such as hematoma that require blood transfusion remain one of the leading causes of morbidity after a PCI. The American College of Cardiology (ACC) defines a significant hematoma as "a collection of blood under the skin measuring $10 \mathrm{~cm}$ or more, with a drop in hemoglobin of three grams, and requires transfusion for treatment with red blood cells or whole blood, and or requires procedural intervention such as surgery at the bleeding site to reverse or stop the bleeding, and or a prolonged hospital stay" (ACC, $2011 \mathrm{pg} 73$ ).

Even though technological advances continue to grow in cardiac catheterization and PCI, vascular complications remains the most common complication with hematoma development after femoral artery puncture being the most frequent (Cosman et al., 2010). Patients who experience hematomas in the groin may experience a decreased quality of life, pain, and often a tingling sensation in that extremity, which has been reported to last for months (Andersen, Bregendahl, Kaestel, Skriver, \& Ravkilde, 2005). 
Research examining vascular complications has focused on many different potential contributors including the size of the sheath, gender, body mass index (BMI), skill of the operator, location of the puncture, the use of Glycoprotein (GP) IIb/IIIa inhibitors, thrombin inhibitors, number of punctures, activated clotting time, method of compression, closure devices, length bed rest, renal failure, practitioners' efficiency, and personnel removing sheath. No one variable or combination has been identified as the significant cause of hematomas (Applegate et al., 2008; Berry, Kelly, Cobbe, \& Eteiba, 2004; Doyle et al., 2007).

Juran, Rouse, Smith, O'Brien, DeLuca, and Sigmon (1999) conducted a landmark descriptive correlational study involving 4010 subjects. The objective of the study was to measure the relationship between nursing interventions and vascular complications at the arterial access site in patients who underwent PCIs. The authors also proposed to recommend a standard of care that minimized bleeding complications. The Juran et al. study was a comprehensive research study that integrated nursing research, physicians, and a pharmaceutical company to develop a nursing sub study group within a company sponsored, clinical trial. The main trial was titled Integrilin to Manage Platelet Aggregation to Prevent Coronary Thrombus (IMPACTII) and the nursing sub study was titled Standards of Angioplasty Nursing Techniques to Diminish Bleeding around the Groin (SANDBAG). The study had two goals. The first goal was to determine the current patterns of nursing practice after PCI. That goal was accomplished by a survey and assessment of standards for patient care at 82 sites participating in IMPACTII study. The second and most significant goal in the SANDBAG study was to implement nursing recommendations based on the evidence of the large, randomized clinical trail.

The SANDBAG study was one of the first studies to acknowledge the effects of evidence based practice on nursing standards, and the effect the changes had on decreasing post PCI 
complications. The study implemented protocols for measuring activated clotting times at the bedside before sheath removal, discontinuing heparin after sheath removal, and decreasing use of heparin infusions in appropriate patients after PCIs. Early sheath removal was noted as a significant factor in decreasing bleeding complications at the arterial site. Results compared earlier sheath pull versus later sheath pull and demonstrated a decrease of $5 \%$ in bleeding complications and a decrease in length of stay by $1.7 \%$. Results demonstrated a bleeding rate of $5.4 \%(n=51 / 941)$ for sheath times $<7.1$ hours as compared to $10.3 \%(n=97 / 943)$ for sheath times $<23.7$ hours. This finding demonstrated that longer sheath times were related to higher bleeding complications (Juran et al., 1999).

Berry et al. (2004) conducted a single correlation observational study over a three month period. The study researched the nature and incidence of arterial puncture complications from coronary angiograms and PCIs in clinical practice. The premise of this project was based on the underreporting of hematomas post coronary angiogram and PCIs, and the substantial amount of hospital expenditures that may arise from these complications. The study calculated sheath times as a variable during the length of the procedures, not the total sheath time. Three hundred eleven consecutive procedures were included, of which 237 were coronary angiograms and 74 were PCI; 309 procedures (99\%) involved femoral arterial access. Data included the use of clopidogrel, low molecular weight heparin, unfractioned heparin, glycoprotein IIb/IIIa inhibitor therapies, aspirin, and blood pressure measurements. A study sheet was attached to admission booklets for the staff to complete on each patient during an eight week period.

The definition of hematoma used was a swelling around the arterial puncture site. Hematomas were categorized as being $<5 \mathrm{~cm}$, or $>5 \mathrm{~cm}$. by using an annotated ruler for measurement. The Pearson Chi-Square test was used to compare categorical data. Univiarate 
and multivariate predictors of bleeding complications were determined by regression analysis. The data illustrated the percentage of hematomas rates for coronary angiogram and. PCIs. Sheath times were longer for PCI's than for coronary angiograms (191+-108 minutes versus 49+-54 minutes) $(\mathrm{p}<0.00001)$. The hematomas recorded for $>5 \mathrm{~cm}$ were $11 \%(\mathrm{n}=8)$ in PCI and $6 \%(n=14)$ in coronary angiograms. Hematomas recorded for $<5 \mathrm{~cm}$ were $31 \%(n=22)$ for PCI and $17 \%(n=40)$ for coronary angiogram. The largest percent of hematomas was for PCIs at 41 $\%(n=73)$, whereas coronary angiograms were calculated at $22 \%(n=56)$. The study alluded more to the variables of female gender, increased BMI, and increased catheter size as the significant risk factors in causing hematomas, rather than sheath times.

Andersen et al. (2005) conducted an audit to examine the incidence of hematomas after coronary angiograms and PCI's via the femoral artery, and identified predictors that increased the risk for developing hematomas. The audit included 474 medical records during a four month period. Three hundred and twenty two subjects underwent coronary angiogram, 141 underwent PCI, and 11 were excluded due to missing data. Approximately $85 \%$ of the procedures were made via the femoral artery, $10 \%$ via the radial, and 5\% the brachial. A hematoma was defined as an accumulation of blood at the skin level with a diameter of $>5 \mathrm{~cm}$ in the area of artery puncture. The hematoma was always assessed by two nurses and/or a nurse and a doctor for reliability. Twelve risk factors were identified among the 33 variables, with the most significant identified as the artery access. The research emphasized the importance of an experienced operator and acknowledged that an experienced operator will also be able to reduce sheath time as well. The study $(n=463)$ demonstrated that the frequency of hematomas was $1.3 \%(n=6)$ that were $>10 \mathrm{~cm}$, and $8.9 \%(\mathrm{n}=41)>5 \mathrm{~cm}$. 
The results were further categorized according to coronary angiogram or PCI. The risk of hematoma for coronary angiograms was $7.5 \%$, and $12.1 \%$ with PCI's. The data was comparable to similar studies, but significantly higher than the American College of Cardiology incidence for complications ( $1 \%$ for coronary angiograms and $3 \%$ for PCI). The risk factors that were identified for developing a hematomas were female, multiple arterial punctures, active clotting time $(\mathrm{ACT})$ time $>175$ seconds, systolic blood pressure $>160 \mathrm{~mm} \mathrm{Hg}$, inexperienced operator, personnel changes during compression, use of 11b-11Ia inhibitors, use of anticoagulation treatment, and sheath time greater than 16 minutes. These risk factors all provide background for changes in nursing procedures. The research provided actual documented data related to the importance of decreased sheath time, and labeled increased sheath time as a possible risk factor for developing hematomas. The data demonstrated a need to focus more care on patients that are at an increased risk for developing hematomas both in the cardiac catheterization lab and on the nursing units. Utilization of the data collected enabled changes to be made to remove sheaths more efficiently in order to decrease the risk for hematomas (Andersen et al., 2005).

Applegate et al. (2008) conducted a single-center observational study to evaluate if strategies to reduce vascular complications from sheath placement at the femoral artery in the past decade have improved. Strategies included the use of fluoroscopy to guide femoral artery access, utilization of vascular closure devices, and the use of smaller sheath size. The study was conducted over a ten year period form 1998-2007. The participants included a total of 35,016 subjects undergoing consecutive diagnostic cardiac catheterization $(n=20,777)$ and PCI $(n=14,239)$. The majority of the procedures $(99 \%)$ were performed via the femoral artery. Annual rates of vascular complications were evaluated. Covariate effects on the risk of vascular 
complications were evaluated by logistic regression and risk-adjusted trend analysis. The study provided significant background information related to complications after coronary angiograms and PCIs. All sheaths were reported as removed soon after the procedure, with no specific time measurement as to how soon a sheath was removed. Guidelines were provided to assist the practitioner to decide when the sheath could be pulled including activated clotting time less than or equal to 180 seconds in subjects who received heparin and greater or equal to 2 hours in subjects who received angiomax. Subjects who did not receive closure devices had the sheath pulled when the activated clotting time (ACT) was less than or equal to 180 seconds. Subjects who received either heparin or bilvalirudin had their sheaths pulled within two hours of the discontinuation of the infusion.

The results of the study noted earlier sheath removal as one of the possible variables that contributed to a significant decrease of vascular complication. Comparing rates from the time periods of 1998 - 2007, catheterization association complications were $1.7 \%$ to $0.2 \%$ respectively and with PCIs from $3.1 \%$ to $1.0 \%$ respectively $(\mathrm{p}<0.001)$. A decrease in hematomas $>10 \mathrm{~cm}$ accounted for the greatest reduction in vascular complication rates: $1.1 \%(\mathrm{n}=47)$ in 1998 to $0.3 \%(n=2)$ in $2007(\mathrm{p}<0.001)$. Results suggest that sooner sheath removal time is a positive factor decreasing vascular complications, and decreased sheath size was beneficial in decreasing vascular complications. Female gender, decrease in body mass index (BMI), and larger sheath size were discussed as co-variates that led to an increase in complication rates. For coronary angiography, the risk of vascular complications was lower for 4-french size as compared to other larger sheath sizes. PCI sheath size of 6-7 french had similar reported risk for vascular complications but risk was less than that for 8 and 10 french sheath sizes. The study provided 
valuable data related to changes in practice that were implemented to aid in hematoma prevention, but a limitation was that specific sheath times were lacking.

Doyle et al. (2008) conducted a quantitative non-experimental study that evaluated major vascular complications in three groups of subjects. A total of 17,901 patients underwent PCIs. Group I $(n=2,443)$ received extensive anticoagulation and antiplatelet therapy before and during PCIs as well as dextran, warfarin, heparin, aspirin and dipyridamole. Group II $(n=6,212)$ received anti platelet combination therapy including aspirin and ticlopidine, and peri procedural (GP) IIb/ IIIa inhibitors. Group III ( $\mathrm{n}=9,253)$ received aspirin and clopidogrel, along with (GP) $\mathrm{IIb} / \mathrm{III}$ inhibitors but at a reduced intensity and duration of the medications.

The study reviewed changes that had occurred in anticoagulation protocols, antiplatelet therapies, and procedure time and evaluated if the changes lead to decreased rates in vascular complications. Hematomas were considered major vascular complications. Defining characteristics of the hematoma were identified as greater than four centimeters in diameter and those that required blood transfusion therapy. The study evaluated procedural characteristics such as reduction sheath size, mean procedure time, and the dosing and duration of anticoagulation with heparin. The study concluded that reduced sheath size, careful monitoring of anticoagulation and platelet inhibitors, along with the practitioners' efficiency led to a decrease of vascular complications. Group I demonstrated an $8.4 \%$ reduction in hematomas $(\mathrm{n}=2,441)$ with 172 femoral hematomas; Group II had a 5.3\% reduction $(\mathrm{n}=6,207)$ with 236 femoral hematomas; and Group III yielded a 3.5\% reduction $(n=9,253)$ with 257 hematomas for vascular complications. Results demonstrated a decrease in vascular complcations of $4.9 \%$ $(\mathrm{n}=17,901)$ across the three groups. Minimized sheath dwell time was described as a contributing factor to the decline in major vascular complications, with the duration of 
procedures time decreasing from 1.7 hours to 1.4 hours. The study provides documentation that decreased sheath time during procedures has a positive affect on decreasing vascular complications, specifically hematomas. The authors infer that decreased sheath time may be beneficial to decreasing risk of hematomas (Doyle et al., 2007).

In conclusion, the importance of early sheath removal has been shown to be beneficial on decreasing hematomas in patients after PCIs. The literature demonstrated an increase risk for developing hematomas in female gender, increased BMI, and large sheath sizes. Smaller sized catheters, increased efficiency of the practitioners, and shorter procedure times were shown to be beneficial in decreasing hematomas. The literature also includes the standardized testing of the activated clothing time and minimal use of thrombin inhibitors in providing criteria for when it is safe to remove a sheath. However, the literature lacks consistent data specific to sheath dwell and removal time.

Next, the theoretical framework used to guide this research project will be introduced. 


\section{Theoretical Framework}

The Theory of Unpleasant Symptoms, originally published in 1995 (Lenz, Suppe, Gift, Pugh, \& Milligan), and then updated in 1997 (Lenz et al.), will be used as the theoretical framework to guide this research. The Theory of Unpleasant Symptoms is a middle range theory, and was developed to guide nursing research and practice across multiple clinical populations. Middle range theories were a change from the earlier grand nursing theories, which had been described more as blueprints for nursing than as potential guidelines for practice. The Theory of Unpleasant Symptoms design was intended to assist nurses in symptom management, with the goal of linking nursing practice to research. The theory proposes that each individual processes an unpleasant symptom in a different manner, and that an individualized plan of care to help prevent the symptoms from occurring is necessary in caring for the patient (Lenz et al., 1995).

Lenz et al. (1997) suggested that the patients 'symptoms are caused by various factors including physiological, psychological, and/or situational factors. The theory requires nursing to evaluate and identify the factors and acknowledge that they are interrelated. Lenz et al. (1995) described the symptoms in terms of duration, intensity, distress, and quality. This theory acknowledges a link between the three factors and how they interact in an individual and may lead to a possible negative affect on the individual's experience. This in turn can lead to alteration in an individual's cognitive, functional, or physical performance.

The Theory of Unpleasant Symptoms was used to guide proposed research study. The study examined the unpleasant symptom of hematomas formation in post PCI patients. Hematomas may be caused by outside situational factors such as increased sheath time, which was the focus of this study. This author proposed that the length of time a sheath remains in 
place may lead to the physiologic process of hematoma development. This in turn causes an increase in the patient's pain and anxiety. Alterations in situational factors may result, such as a delay in their ability to return to work, or home, and an alteration in current role. The most common residual effects of the hematoma were inability to walk normal and unpleasant tingling sensation in the leg (Andersen et al., 2005). Hematomas impact the patient's well being, and in many cases may be preventable after PCI. 


\section{Methodology}

\section{Purpose}

The purpose of this study was to determine if there was an increased incidence of hematomas in individuals undergoing percutaneous coronary intervention with associated sheath times of greater than five hours as compared to sheath time of less than five hours. It was proposed that those individuals whose sheath time was greater than five hours would have a higher risk for developing a hematoma.

\section{Research question}

The research question was: Does prolonged femoral sheath time of greater than five hours increase a patients' risk for developing a hematoma formation after a PCI?

\section{Design}

The project employed a retrospective two group design. Group A (control) included subjects who met the inclusion criteria and had their femoral sheath removed within five hours of insertion. Group B (experimental group) included subjects who met the inclusion criteria and had the femoral sheath removed greater than five hours from time of insertion.

\section{Site}

The data collection took place at a tertiary care center in Providence, Rhode Island. The research hospital is a private, not for profit hospital that is licensed for 247 patient beds. The research hospital is a major teaching affiliate of the Warren Alpert Medical School of Brown University specializing in Cardiac Care. The research hospital performed approximately 1500 PCIs last year. 


\section{Sample}

The sample was recruited from the data base of patients who have had PCIs completed at the research hospital. Inclusion criteria included subjects over the age of 18 who required an elective PCI procedure following a cardiac catheterization. Exclusion criteria included procedures not accessed via the femoral artery, patients with known bleeding disorders, and multiple puncture attempts to the artery, those who developed an immediate hematoma, emergent PCI procedure, and also hemodynamic instability before, during, or after the PCI.

\section{Measurement}

A data collection tool (Appendix A) was designed by the student researcher based on the literature and clinical experience. After IRB approval, the tool was pilot tested to assure completeness and usability. Basic demographic data and data specific to the PCI were collected.

\section{Procedures}

Upon approval by the Lifespan and Rhode Island College IRB's, the researcher obtained a list of all potential subjects who had undergone a PCI between January and June of 2012 at the study site and with a medical record ICD-9 (International Classification of Diseases Codes, 2010) of V4582. The list was obtained from the quality control and assurance coordinator manager. No personal health information collected was electronically linked, and the list was destroyed upon completion of the data collection. De-identified data were entered into an excel spread sheet. All data obtained was stored in a locked file to which only the researcher and faculty advisor had access to, and confidentiality of all data was maintained.

Data were collected by the student researcher. Approximately 250 medical records were reviewed to obtain a cohort of 55. Data were obtained from the documentation in the patients' cardiac catheterization record/medical record. Documentation of a hematoma was based on the 
definition of the American College of Cardiology (ACC). The ACC defines a hematoma "as a collection of blood under the skin measuring 10 centimeters or more, with a drop in hemoglobin of three grams, and requires transfusion for treatment of red blood cells, and or requires procedural intervention such as surgery at the bleeding site to reverse or stop the bleeding" (ACC, 2011, p. 73). Upon review of the medical records, it was identified that actual measurement of hematomas post procedure was inconsistent. Documentation of hematomas was limited to descriptive data, such as using the terms "small", "medium," and "large" to describe the size Recognizing that the institution's official counting of hematoma incidence was limited to the ACC definition, the student researcher determined that hematomas documented in the record that did not meet this definition were of interest to nursing practice. For the purpose of attempting to quantify these hematomas, the student researcher designated a hematoma documented as "small" to be $2 \mathrm{~cm}$. in size, a "medium" hematoma as $4 \mathrm{~cm}$, and "large" $8 \mathrm{~cm}$.

\section{Data Analysis}

Descriptive statistics were used to analyze the study variables, including frequencies, percentages, and mean scores. Data were analyzed and compared by group, and group scores were further analyzed by gender. Femoral sheath times greater than 5 hours versus sheath times less than 5 hours and the use of certain medications IIb/IIIa glycoprotein inhibitors; heparin, low molecular heparin, and thrombin inhibitors were analyzed regarding hematoma formation post PCI procedures. 


\section{Results}

A total of 250 medical records were reviewed to obtain a cohort of 55 subjects who underwent PCIs. Group A, those individuals whose sheaths were in place for less than five hours, included 24 subjects; Group B, individuals whose sheath were in place for greater than five hours, included 31 subjects. Exclusions included such factors as radial artery access, closure device usage within the femoral artery, and or hemodynamicaly instability patients before, during, or after the procedure.

Demographic data by gender are listed in Table 1. Data are presented by gender since gender is identified as a risk in the literature, with females at increased risk of hematoma post procedure. Increased or decreased BMI for all genders has also been identified in the literature. Table 1

Demographics Data by Gender

\begin{tabular}{|c|c|c|c|c|c|}
\hline Gender & Number & $\begin{array}{c}\text { Average } \\
\text { Age }\end{array}$ & $\begin{array}{c}\text { Average } \\
\text { Height } \\
\text { (cm) }\end{array}$ & $\begin{array}{l}\text { Average } \\
\text { Weight(kg) }\end{array}$ & $\begin{array}{c}\text { Average } \\
\text { BMI }\end{array}$ \\
\hline Male & 34 & 65 & 174 & 90 & 31 \\
\hline Female & 21 & 68 & 159 & 73 & 29.3 \\
\hline
\end{tabular}

The majority of the subjects were male. Both genders had high BMI rates at $29-30 \%$ above target. Average ages of the subjects were 65 years for males and 68 years for females'. Recommended BMI for adults $>20$ years of age is less than or equal to $25 \mathrm{~kg} / \mathrm{m} 2$ (AHA, 2012).

Six French sheath size was used for all of the procedures except one. Figure 1 illustrates the ratio of male to female gender for each group in relation to the average length of time the femoral sheaths were in place. 


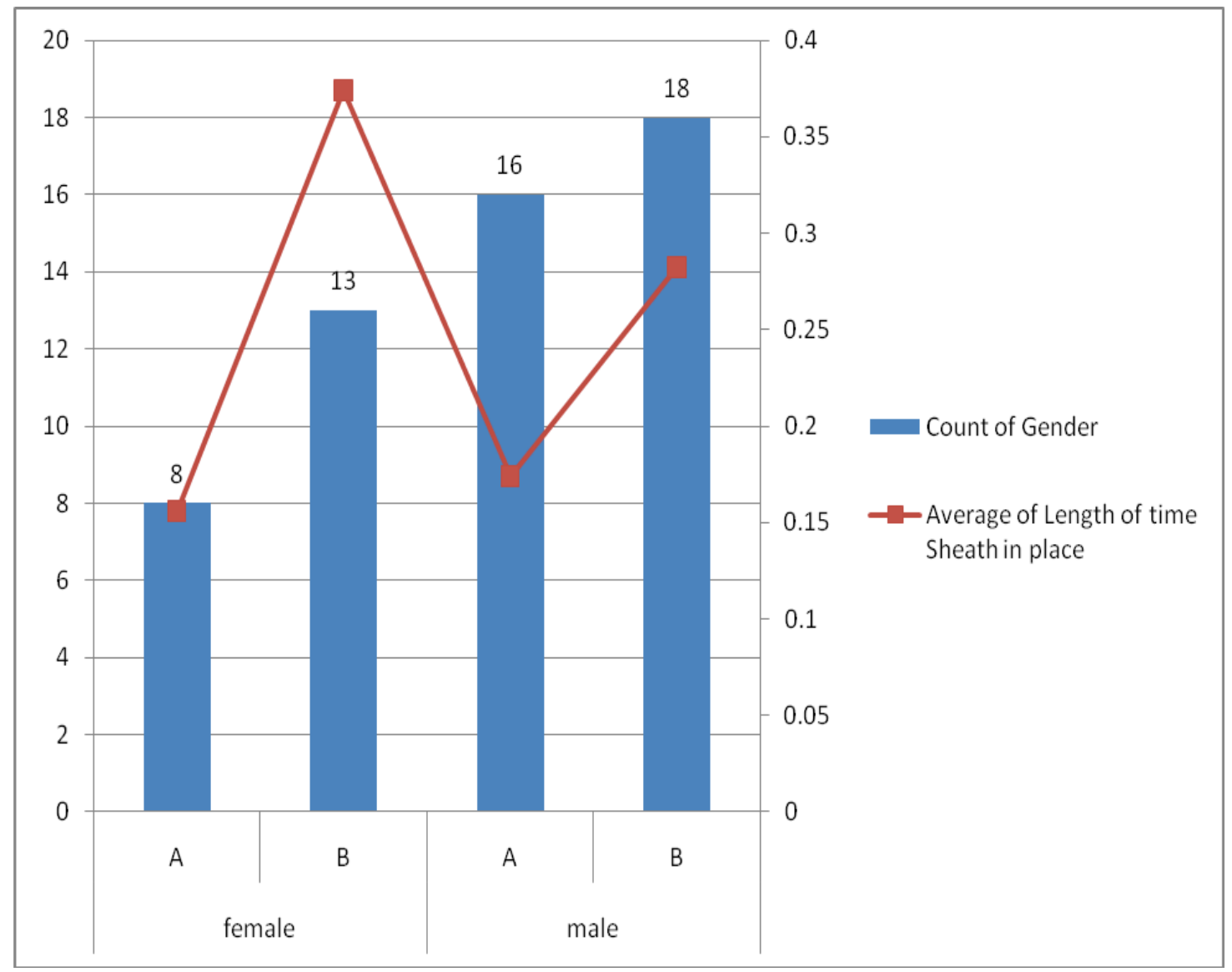

Figure 1. Average length of time sheaths in place in relationship to Group A and Group B by gender.

There were 16 males and eight females in Group A, and 18 males and 13 females in Group B. The range of sheath time for Group A was 1.49 to 4.55 hours, with a mean sheath time of 4.02 hours. Group B sheath time range was 5.07 to 13.33 hours, with a mean of 7.42 hours. No hematomas were identified using the ACC definition, which was the measurement standard for this research hospital. The reported hematomas were lower than the national averages and the majority of the literature reviews noted in the study. Seven charts of the $55(12 \%)$ included descriptive documentation of hematomas by sheath removers who were nurse practitioners or cardiovascular technicians. These were characterized in the record as "small", "medium", and 
"large". One hematoma was identified by the researcher as an outlier and was not included in the data collection results. Table 2 illustrates the characteristics of the remaining six hematomas as well as the coagulation-impacting medications received by these subjects during the procedures. The yellow shading illustrates those subjects who received both thrombin inhibitor and heparin.

Table 2

Descriptive Hematomas by Gender, Sheath Time, and Coagulation-Impacting Medications

\begin{tabular}{|l|l|l|l|l|l|l|}
\hline $\begin{array}{l}\text { Subjects } \\
\text { M=male } \\
\text { F=Female }\end{array}$ & $\begin{array}{l}\text { Sheath } \\
\text { times }\end{array}$ & $\begin{array}{l}\text { Hematoma } \\
\text { Size }\end{array}$ & $\begin{array}{l}\text { Low } \\
\text { molecular } \\
\text { Heparin }\end{array}$ & Heparin & $\begin{array}{l}\text { Thrombin } \\
\text { inhibitor }\end{array}$ & $\begin{array}{l}\text { Glycoprotein } \\
11 \text { b-111a } \\
\text { inhibitor }\end{array}$ \\
\hline $1-\mathrm{M}$ & 5.39 & $2.0 \mathrm{~cm}$. & No & Yes & Yes & No \\
\hline $2-\mathrm{M}$ & 5.43 & $2.0 \mathrm{~cm}$. & No & No & Yes & Yes \\
\hline $3-\mathrm{M}$ & 7.13 & $2.0 \mathrm{~cm}$. & No & Yes & No & Yes \\
\hline $4-\mathrm{F}$ & 8.10 & $8.0 \mathrm{~cm}$. & No & Yes & Yes & No \\
\hline $5-\mathrm{M}$ & 10.34 & $2.0 \mathrm{~cm}$. & No & Yes & No & Yes \\
\hline $6-\mathrm{F}$ & 10.47 & $4.0 \mathrm{~cm}$. & No & Yes & No & Yes \\
\hline
\end{tabular}

Four $(11 \%)$ males and two females (9\%) subjects developed hematomas. Fifty percent of the six hematomas described in the record $(n=3)$ received the thrombin inhibitor, angiomax, $66 \%$ received a glycoprotein IIb-IIIa inhibitor -plavix $(n=4)$, and $83 \%(n=5)$ received intravenous heparin. The student researcher converted the documented hematomas from the chart as: "small" = 2cm; "medium" $=4-6 \mathrm{~cm}$; and "large" $=8 \mathrm{~cm}$. Ten centimeters was not utilized as a description of large hematomas due to the fact that this would have qualified the patient for the ACC definition of hematoma and none of the patients were classified as having reportable hematomas.

Greater than $80 \%$ of both groups $(\mathrm{n}=55)$ received heparin and a glycoprotein IIb-IIIa inhibitor, with group B being the highest at $94 \%(n=17)$ for the males and $92 \%(n=12)$ for females. Thrombin inhibitors were utilized in both groups, with female subjects receiving the 
least $(12 \% ; n=1)$ in group A, and 7.6\% $(n=1)$ in Group B. Males received thrombin inhibitors at a higher rate $(25 \% ; n=4)$ in Group A and Group B $(27.7 \% ; n=5)$. Low molecular weight heparin was not used in group A, and was used in low rates in Group B $(7.6 \% ; n=1$ females $)(5.5 \% ; n=1$ males $)$.

To further analyze contributors to prolonged sheath time, those subjects who were transferred from another facility were identified. Group A had one subject transferred from another facility (2.4\%), with a sheath time of 3.50 hours; the remaining subjects in Group A who were not transferred from another facility had an average sheath times of 3.5 hours $(n=23 ; 95$ \%). In Group B, eight subjects (33\%) had been transferred from other facilities, with an average sheath time of 9.29 hours, while those in Group B who were not transferred had an average sheath time of 6.9 hours $(n=23 ; 74 \%)$. 


\section{Summary and Conclusions}

Cardiac disease remains the number one cause of death in persons over the age of 75 , and is ranked the number two cause of death in persons aged 25-74. Advancement in technology and medicine has lead to a significant increase in the number of cardiac catheterizations and PCIs performed in the past 15 years (Shoulders-Odom, 2008). There were over 1,300,000 PCI procedures performed in the US in 2006 (AHA, 2009). Hematomas are a complication that may occur as a result of these procedures. The literature review provided data documenting a positive correlation between female gender, renal insufficiency, low BMI, high BMI, the use of certain thrombin inhibitors, and glycoprotein IIb/IIIa inhibitors as contributors to an increased risk factor in developing hematomas (Berry et al., 2004). No single variable has been identified as the primary contributor to, nor has the literature directly identified sheath times as a causative factor.

The purpose of this study was to determine if there was an increased incidence of hematomas in individuals undergoing percutaneous coronary intervention with associated sheath times of greater than five hours as compared to sheath time of less than five hours. It was proposed that those individuals whose sheath time was greater than five hours would have a higher risk for developing a hematoma. The study was guided by the theory of The Theory of Unpleasant Symptoms. A retrospective chart review was conducted; 250 charts were reviewed of which 55 met the inclusion criteria. Group A $(n=24)$ included subjects whose sheath time was less than or equal to five hours. Group B $(n=31)$ were subjects whose sheath time was greater than five hours. The data demonstrated a $0 \%$ hematoma rate in both groups per the ACC criteria. The rate of complications for diagnostic catheterization has been cited at $1 \%$ and $3 \%$ for PCI's, but vascular complications alone have been reported from $0.1 \%$ up to $61 \%$, depending on 
the definition of complications and covariates (Dumont, Keeling, Bourguignon, Sarembock, \& Turner, 2006).

Six descriptive documented hematomas were noted that did not meet the ACC criteria for hematoma. The six hematomas all had sheath times of greater than five hours, suggesting a potential relationship between prolonged sheath time and hematoma development. In contrast to the literature, female gender was not found to be a contributing risk factor; the majority of documented hematomas occurred in males $(n=4 ; 66 \%)$. Increased BMI was characteristic in both groups, consistent with the literature review. Fifty percent of the six hematomas described in the record $(n=3)$ received the thrombin inhibitor, angiomax, 66\% received a glycoprotein $\mathrm{IIb} / \mathrm{III}$ inhibitor -plavix $(\mathrm{n}=4)$, and $83 \%(\mathrm{n}=5)$ received intravenous heparin. The literature review supported that the use of thrombin inhibitors and IIb/IIIa glycoprotein inhibitors as contributing factors in the development of hematomas.

The student researcher speculated that the majority of individuals in Group B would have been transferred from other hospitals and would have subsequently had the PCI completed at the research hospital. However, only $25 \%(n=8)$ of those with sheath times of greater than five hours were transfers. The majority of the patients started and completed their PCI's at the research hospital. This suggests that organizational factors contribute to the prolonged sheath dwell times. As suspected, the majority of patients in group A began and completed their PCI's at the research hospital $(\mathrm{n}=1 ; 4 \%)$.

The study was limited by factors associated with a retrospective design. In particular, documentation in the medical records lacked reliability in regard to the manner in which hematomas were subjectively described. A standard, reliable, and valid measure of hematoma size was not used to document hematoma size. The study was also limited by the small sample 
size. No attempt was make to collect other demographic data including race and ethnicity, so the impact of such variables cannot be identified.

In this study, all six subjects who experienced hematomas had sheath time of greater than five hours. Clearly, further study is indicated, also supported by the fact that actual documentation of sheath times in the literature is scant. Results documented that those subjects who did experience hematoma had also received, IIb/IIIa glycoprotein inhibitors, and thrombin inhibitors. No attempt was made to examine this relationship in subjects who did not develop a hematoma, and no conclusions can be drawn. Hematomas after cardiac catheterization and PCI can produce significant, negative outcomes for patients, and further nursing research related to prevention, early detection, and management is needed. 


\section{Recommendations and Implications for Practice}

Advanced Practice Registered Nurses (APRNs) are called upon to lead by example and provide high quality nursing care. Prevention and management of post cardiac intervention complications, including hematomas, is an important pursuit. Specific to the findings in this study, APRNs can be instrumental in driving the development and implementation of evidence based protocols that include accurate documentation of hematomas. Methods for assessing, recording, and documentating hematomas must be clearly specified and evidence based.

Evidence based documentation drives practice improvement, and would be beneficial to both the institution and the patients receiving care. Using a ruler is a simple, inexpensive, and validated tool that should be included as standard of care for documentation of any hematoma. Documentation of skin alterations is mandated by the Centers for Medicare and Medicaid Services (CMS), including size and descriptive adjectives that may be useful in defining and classifying the alteration. A hematoma by definition is a collection of blood under the skin, and should be documented in the same manner as any other skin alteration.

APRNs can continue to drive practice and policy change in their work place by being recognized leaders in such areas as evidence based practice councils and inter-disciplinary teams. APRN's working in cardiac catheterization labs and cardiology should lobby to promote a standardized documentation method for hematoma measurements. APRNs, as role models for practicing nurses, are in an excellent position to educate and demonstrate bedside nurses about the importance of hematoma assessment and accurate documentation.

Further practice change and research is needed related to the length of time a sheath remains in place. In addition to hematoma development, prolonged sheath time is associated 
with all of the risks of immobility or prolonged bed rest, including increased pain, pressure ulcers, atelectasis, deep vein thrombosis, and difficulty voiding. Prolong sheath time increases a patients' exposure to all of the complications associated with immobility and can also lead to an increased length of stay. APRNs should work with members of the inter-disciplinary team to examine organizational/systems issues that contribute to prolonged sheath times. Once identified, and with needed administrative support, strategies to minimize modifiable factors can be implemented and evaluated. Though it is not clear what the 'ideal' sheath dwell time is, the literature clearly supports that withdrawal as soon as is clinically feasible is indicated. It is hypothesized that this is not a simply an issue at the study site; thus, lobbying for practice change at the national level through professional nursing organizations is warranted. With a continuous increase in cardiac laboratories and advancements in interventional cardiology, these actions are necessary to ensure safety for our patients.

Additional research related to the identification of minimal, ideal sheaths times is needed. In the literature, sheath times have been recorded from as little as 16 minutes and is this study sheath times of 1.49 hours to 13.33 hours were documented. Prolonged sheath times when not clinically indicated are detrimental to quality patient care and need to be addressed through advanced nursing practice, education, policy change, and on-going research. 


\section{References}

Alderman, E., Kip, K., Whitlow, P., Bashore,T., Fortin,D.,Bourassa, M.,Lesparance, J.,...Schwartz, L. (2004). Native coronary disease progression exceeds failed revascularization as cause of angina after five years in the bypass angioplasty revascularization investigation (BARI). Journal of the American College of Cardiology, 4(18), 766-774.

American College of Cardiology Foundation. (2011). NCDR ${ }^{\circledR}$ cathPCI registry ${ }^{\circledR}$ v4.4.

Retrieved from http://www.cardiologyinoregon.org/2012_NCDR_in_Oregon/CathPCI_v4_CodersDictio nary 4.4.pdf

American Heart Association, (AHA). (2009). Heart Disease \& Stroke Statistics-2009 Update.Dallas, Texas: Retrieved from: doi:10.1161/CIRCULATIONAHA.108.191261

American Heart Association, (AHA). (2012). Heart Disease and Stroke Statistics 2012-Update Dallas, Texas: Retrieved from: http://circ.ahajournals.org/by guest June 21, 2012

Andersen, K., Bregendahl, M., Kaestel, H., Skriver, M., \& Ravkilde, J. (2005). Hematoma after coronary angiography and percutaneous coronary intervention via the femoral artery frequency and risk factors. European Journal of Cardiovascular Nursing, 4, 123-127.

Applegate, R., Sacrinty, M., Kutcher, M. , Kahl, F., Gandhi, S., Santos, R., \& Little, W. (2008). Trends in vascular complications after diagnostic cardiac catheterization and percutaneous coronary intervention via the femoral artery 1998-2007. JACC: Cardiovascular Interventions, 1, 319-326. doi: 10.1016/j.jcin.2008.03013 
Berry, C., Kelly, J., Cobbe, S., \& Eteiba, H. (2004). Comparison of femoral bleeding complications after coronary angiography versus percutaneous coronary intervention. The American Journal of Cardiology, 94, 361-363. doi: 10.10.1016/j.amjcard.2004.04.036

Cale, L., \& Constantino, R. (2012). Strategies for decreasing vascular complications in diagnostic cardiac catheterization patients. Dimensions of Critical Care Nursing, 31(1), 13-17. doi: 10.1097/DCC.0b013e31823a52f5

Cosman, T., Arthur, H., \& Natarajan, M. (2011). Prevalence of bruising at the vascular access site one week after elective cardiac catheterization or percutaneous coronary intervention. Journal of Clinical Nursing, 20, 1349-1356.. doi: 10.1111/j.136-2702.2010.03595.x

Doyle, B., Ting, H., Bell, M., Lennon, R., Matthew, V., Singh, M., Holmes, D., \& Rihal, C. (2007). Major femoral bleeding complications after percutaneous coronary intervention. JACC: Cardiovascular Interventions, 1(2), 202-209. doi:10.1016/j.jcin.2007.12.006

Dumont, C., Keeling, A., Bourguignon, C., Sarembock, I., \& Turner, M. (2006). Predictors of vascular complications post diagnostic cardiac catheterization and percutaneous coronary interventions. Dimensions of Critical Care Nursing, 25(3), 137-142.

Girotra, S., \& Cram, P. (2012). Universal access to a percutaneous coronary intervention hospital is it feasible or desirable? Circulation Cardiovascular Quality Outcomes, Retrieved from htp://ciroutcomes.ahajournals.org

Juran, N., Rouse, C., Smith, D., O’Brien, M., Deluca, S., \& Sigmon, K. (1999). Nursing intervention to decrease bleeding at the femoral access site after percutaneous coronary intervention. American Journal of Critical Care, 5(5), 303-313. 
Lenz, E.R., Suppe, F., Gift, A., Pugh, L., \& Milligan, R. (1995). Collaborative development of middle range nursing theories: Toward a theory of unpleasant symptoms. Advance Nursing Science, 17(3), 1-13.

Lenz, E. R., Suppe, F., Gift, A., Pugh, L., \& Milligan, R. (1997). The middle-range theory of unpleasant symptoms: An update. Advances in Nursing Science, 17(3), 14-27.

Libby, P., \& Theroux, P. (2005). Pathophysiology of coronary artery disease. Circulation, 111, 3481-3488. doi:10.1161/CIRCULATION AHA.105.537878

Lichtenstien, A., Appel, L., Brands, M., Carnethon, M., Daniels, S., Franch, H., Franklin, B., ... Howard, B. (2006, June 19). Diet and lifestyle recommendations revision 2006: A scientific statement from the American Heart Association Nutrition Committee. Circulation, 114(1), 82-96. doi: 10.1161/CIRCULATIONAHA.106.176158

Lloyd-Jones, D., Adams, RJ., Brown, TM., Carnethon, M., Dai, S., DeSimone, G., Ferguson, TB., Ford. E.,... Gillespie, C. (2010). Executive summary: heart disease and stroke statistics - 2010 update a report from the American Heart Association. Circulation 121(7), e47-e202.doi:10.1161/CirculationAHA.109.192666

McConnell, M., McDilda, K., Bridges, R., Marsh, N., Jenkins, G., Dowdy, J., \& Prasnikar, M. (2012). Comparison of different methods for achieving hemostais after arterial sheath removal. Journal of Cardiovascular Nursing, 27(4), E1-E5.

National Center for Health Statistics. (2010). Rhode Island fact sheet, National vital report sheet, 59(10). Washington, DC: U.S. Government Printing Office

Patel, M., Jneid, H., Derdeyn, C., Klien, L., Levine, G., Lookstien, R., White, C., ... Rosenfield , K. (2010). Arteriotomy closure devices for cardiovascular procedures: A scientific statement from the American Heart Association. Circulation, 122, 1883-1893. 
doi:10.1161/cir.obo13e3181f9b345 Retrieved from htpp://circ.ahajournals.org/byguest on July 17,2012

Rosamond, W., Flegal, K., Furie. K., Go. A., Greenlund. K., Haase. N., Hailpern. SM.,...Ho, M. (2008). Heart disease and stroke statistics - 2008 update a report form the American Heart Association Statistics Committee and Stroke Statistics Subcommittee. Circulation, 117(4), e25-e146 doi: 10.1161CIRCULATIONAHA.107.187998

Shoulders-Odom, B. (2008, October). Management of patients after percutaneous coronary intervention. Critical Care Nurse; 28(5), 26-40.

Smith Jar, S., Allan, J., Blair, S., Bo now, R., Brass, L., Fonarow, G., Hiratzka, L., ... Krumholz, H. (2006). AHA/ACC guidelines for secondary prevention for patients with coronary and other artherosclerotic vascular diseases: 2006 update: endorsed by the National Heart, Lung, and Blood Institute. Circulation, 113, 2363-2372.

doi:10.1161/CIRCULATIONAHA.106.174516

State of Rhode Island Department of Health. (2011). (Fact Sheet). Providence Rhode Island. Retrieved from http://www.health.ri.gov/

U.S. Department of Health and Human Services. (2008). The seventh report of the joint national committee on Prevention, detection, evaluation, and treatment of high blood pressure (Report JNC 7 Express). Bethesda, MD: National Heart, Lung, and Blood Institute. US Census Bureau. (2010). 2010 Population interactive population search. Retrieved from US Census Bureau: https://census.gov./2010census/popmap/ipmtext.php?fl=44 


\section{Appendix A}

Data Collection Tool

\begin{tabular}{|c|c|}
\hline \multicolumn{2}{|l|}{ Age } \\
\hline \multicolumn{2}{|l|}{ Gender } \\
\hline \multicolumn{2}{|l|}{ Height } \\
\hline \multicolumn{2}{|l|}{ Weight } \\
\hline \multicolumn{2}{|l|}{$\begin{array}{l}\text { Time femoral sheath is } \\
\text { placed }\end{array}$} \\
\hline \multicolumn{2}{|l|}{$\begin{array}{l}\text { Time cardiac catheterization } \\
\text { (CC)completed }\end{array}$} \\
\hline \multicolumn{2}{|l|}{ Is patient a transfer patient? } \\
\hline \multicolumn{2}{|l|}{$\begin{array}{l}\text { Time patient arrived for } \\
\text { percutanous coronary } \\
\text { intervention }(\mathrm{PCI})\end{array}$} \\
\hline \multicolumn{2}{|l|}{ PCI start time } \\
\hline \multicolumn{2}{|l|}{ PCI end time } \\
\hline \multicolumn{2}{|l|}{ Sheath Size (Fr) } \\
\hline \multicolumn{2}{|l|}{$\begin{array}{l}\text { Time femoral Sheath } \\
\text { Removed }\end{array}$} \\
\hline \multicolumn{2}{|l|}{$\begin{array}{l}\text { Length of time Sheath was } \\
\text { in place }\end{array}$} \\
\hline \multicolumn{2}{|l|}{$\begin{array}{l}\text { Hematoma present - per } \\
\text { American College of } \\
\text { Cardiology - NCDR } \\
\text { Definition - Yes/No }\end{array}$} \\
\hline \multicolumn{2}{|l|}{$\begin{array}{l}\text { Hematoma Measurement in } \\
\text { Centimeters }\end{array}$} \\
\hline \multicolumn{2}{|l|}{$\begin{array}{l}\text { Low Molecular Weight } \\
\text { Heparin - Yes/No }\end{array}$} \\
\hline \multicolumn{2}{|l|}{ Heparin - Yes/No } \\
\hline \multicolumn{2}{|l|}{$\begin{array}{l}\text { Thrombin Inhibitors - } \\
\text { Yes/No }\end{array}$} \\
\hline $\begin{array}{l}\text { Glycoprotein IIb-IIIa } \\
\text { Inhibitors - Yes/No }\end{array}$ & \\
\hline
\end{tabular}

